\title{
A GENERALIZATION OF PÓLYA'S INEQUALITY TO STOLARSKY AND GINI MEANS
}

\author{
C. E. M. Pearce, J. PeČArić AND J. ŠUnde
}

\begin{abstract}
Pólya's inequality has recently been extended in a general way involving two geometric means. We show that further extensions to Stolarsky and Gini means are possible. The two occurrences of the mean in each inequality can involve different parameters. Corresponding discrete results are also derived.
\end{abstract}

Mathematics subject classification (1991): 26D15.

Key words and phrases: Inequality between means, Stolarsky means, Gini means, Pólya's inequality.

\section{REFERENCES}

[1] H. Alzer, An extension of an inequality of G. Pólya, Buletin Instittutilui Polytechnic Din, Iasi. Tomal 36(40) Fasc. 1-4 (1990), 17-18.

[2] Z. DARócZY AND L. LosoncZI, Über den Vergleich von Mittelwerten, Publ. Math. Debrecen 17 (1970), 289-297.

[3] C. GinI, Di una formula comprensiva delle medie, Metron 13 (1938), 3-22.

[4] E. Leach AND M. Sholander, Extended mean values II, J. Math. Anal. Appl. 92 (1983), 207-223.

[5] Zs. PÁLES, Inequalities for sums of powers, J. Math. Anal. Appl. 131 (1988), 265-270.

[6] _ Inequalities for differences of powers, J. Math. Anal. Appl. 131 (1988), 271-281.

[7] Comparison of two variable homogeneous means, General Inequalities, (Oberwolfach 1990), (ed. W. Walter), Birkhäuser Verlag, Basel-Boston-Stuttgart 6 (1992), 59-70.

[8] G. PólYA AND G. SzEGÖ, Aufgaben und Lehrsätze aus der Analysis I,II, Springer, Berlin, 1925. 